\title{
Development of a novel platform for quantifying age-related sensori-motor degradation to control age-related falls
}

\author{
G. Shan ${ }^{1} \&$ X. Zhang ${ }^{2}$ \\ ${ }^{1}$ Department of Kinesiology, University of Lethbridge, Canada \\ ${ }^{2}$ Department of PE, Xinzhou Teachers' University, China
}

\begin{abstract}
As the population ages, falls are becoming a major health problem, not only for those with some degree of balance/mobility impairment, but also among healthy active seniors. Previous studies suggest that the degradation of human sensorimotor function related to age contributes to falls. The aim of this research was to develop a practical solution for quantifying the age-related sensori-motor degradation. Therefore, a new testing apparatus, the Dynamic Balance Testing Platform (DBTP), was developed for quantifying sensori-motor decline using Artificial Neural Network (ANN) modeling. The DBTP consists of two layers an immobile base and a mobile perturbation layer. The perturbation layer is balanced on a single center pivot; each of the four corners is supported by an air cylinder, with vertically downwards by a maximum of $2.5 \mathrm{~cm}$. The platform was designed to reflect the natural oscillations in a subject's center of gravity (COG). Shifts in COG positions altered the load on each of the four supporting air cylinders, leading to measurable vertical displacements. The DBTP captures the subject's ability to balance dynamically on a single point of support. An ANN model, trained by 60 subjects' data (age: 18-65+), was developed for the DBTP. Seniors with and without Tai-Chi training were used to contrast any influence from Tai-Chi on the sensori-motor aging. The result demonstrated that Tai-Chi slowed down the effects of sensori-motor aging. This study validated that DBTP is a feasible tool in aging exercise studies.

Keywords: sensori-motor, degradation, ANN modeling, COG, dynamic balance, training effects.
\end{abstract}




\section{Introduction}

Falls and fall-related injuries increase with age. As the population ages, falls become one of the major health problems, not only for those with some degree of balance or mobility impairment, but also among healthy active seniors $[1,2]$. Due to sensori-motor degradation related to age, one third of people 65 years and older experience at least one fall each year [3]. Numerous studies have revealed that falls are the leading cause of external injury among the elderly, accounting for $24 \%$ of emergency visits [4] and $70 \%$ of accidental deaths in persons aged 75 years and older [5]. This severe problem has a significant impact on personal health and healthcare-costs in older adults [6]. As the baby-boom generation enters into retirement age and the mean age of our society rapidly increases, falls and the increased healthcare costs associated with this problem will become a major concern in our society. Developing fall prevention strategies based on an individual sensori-motor function assessment are becoming highly desirable.

Numerous methods for researching the prevention of falls and age-related sensori-motor degradation have been proposed and tested. Current methods for assessing sensori-motor function are based on the analyses of data collected across differing biomechanical tests, such as Static Centre of Pressure (COP) Measurement [7, 8], Dynamic Posturography [9, 10], electromyography (EMG) and motion capture [11, 12]. These tests are often contradictory [13, 14]. Additionally, some approaches are too impractical to use with seniors [15] or too expensive for practitioners [16].

The main reason for the contradictions and/or complexity in existing fallprediction methods is the lack of consideration for the non-linear and redundant interactions amongst afferents and efferents of the human sensori-motor system. Non-linearity arises from neurons receiving impulses from several of their dendrites simultaneously, but limiting their outputs to a single signal. The inputs do not add together in a linear fashion to produce an output of equal magnitude. Redundancy refers to the fact that the human body has a vast quantity of sensory afferents that overlap and there are more sensory inputs than necessary for motor control. This leads to interactions not only amongst afferents and efferents, but also afferents themselves (i.e. afferents affect not only the efferents but also each other). The complex nature of the interactions of sensory neurons influences the sensori-motor system in ways that render accurate predictions based on traditional methods (without the consideration of inputs dependence) difficult if not impossible to achieve. To overcome these difficulties, we initiated an innovative approach using Artificial Neural Network (ANN) modeling, which considers non-linearity and redundancy [17]. We hypothesized that a randomlyperturbed balancing test data, evaluated by ANN modeling would have great potential to become a simple, reliable test for practitioners in the healthcare industry. 


\section{Method development}

Most falls occur from disturbances in balance control mechanisms, or perturbations. For example, when subjects close their eyes while standing still, their body must adjust or re-weight the sensory input to maintain balance. If their body cannot accommodate for the change in sensory input, they may fall. Disturbances in balance stem mainly from vision changes and the supporting base upon which subjects stand. Hence, the re-weighting of the sensori-motor system during instability plays an important role in falls. For these reasons, tests for the characterization of sensori-motor function should not be taken under static conditions, but should instead include measurements taken during perturbations.

We designed, along with operating software, a novel test device - Dynamic Balance Testing Platform (DBTP) - which utilizes an ANN model to evaluate sensori-motor functions. A major advantage of the model lies in its ability to address complications that have made the nervous systems difficult to simulate. These complications include afferent-afferent interactions (i.e. the inputs of a single neuron are not independent but rather interdependent), sensory redundancy (i.e. not all inputs are needed; some inputs can be removed without notable consequences) and neural non-linearity (i.e. the output is not linearly based on the input; biological neurons almost always process a set of multiple inputs for each single output).

\subsection{Dynamic balance testing platform}

Figure 1 displays the design (top) and the prototype (bottom) of the innovative DBTP, which was developed in the Biomechanics Lab at the University of Lethbridge. DBTP consists of two layers - an immobile layer (base) and a mobile layer (perturbation layer). The base is made of a 3/16 sheet of steel measuring $80 \times 80 \mathrm{~cm} 2$ and the perturbation layer of a 3/8 steel sheet (60x60 $\mathrm{cm} 2$ ). The perturbation layer is balanced on a center pivot; each of the four corners is supported by an air cylinder, which can be displaced vertically downwards by a maximum of $2.5 \mathrm{~cm}$. To stabilize the top layer for the mounting and dismounting of subjects, three linear actuators are used. The perturbation layer of the platform was designed to reflect the natural oscillations in subjects' center of gravity (COG). Random shifts in COG positions alter the load on each of the four supporting air cylinders, leading to measurable vertical displacements; the greater the COG changes, the greater the vertical displacements. Essentially, the DBTP captures subjects' attempts to balance dynamically on a single point of support. Thus despite the artificial condition under which the measurements are attained, genuine re-weighting and/or compensatory strategies of the center nerve system (CNS) are reflected and the measurements can be utilized to characterize the sensori-motor functions of a subject. Subjects who introduce greater perturbation to the platform are likely to have less dynamic balance ability. The applied funding will help to evaluate the reliability, possible drawbacks and improvement of DBTP at application level. 

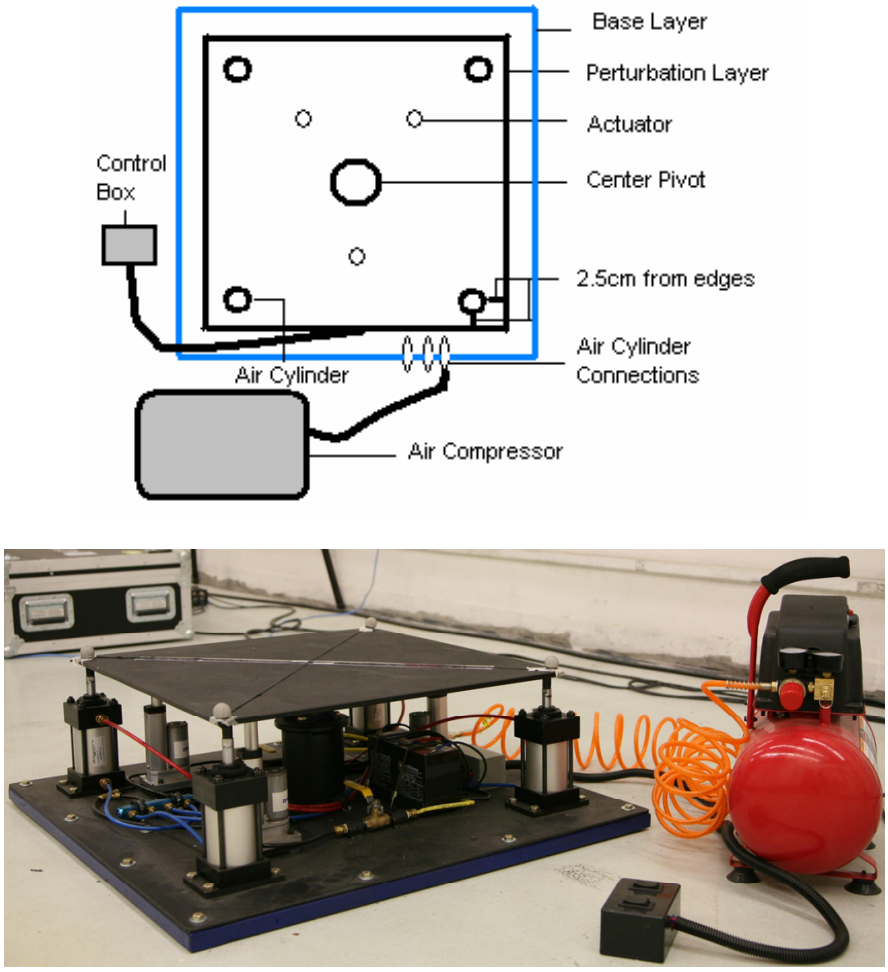

Figure 1: $\quad$ Dynamic Balance Testing Platform (DBTP).

\subsection{ANN modeling}

The ANN model that powers the DBTP is designed to contain three layers (an input layer, a hidden layer and an output layer). The input layer consists of 19 different parameters related to sensory inputs. They include body weight (BW), body height $(\mathrm{BH})$, and parameters derived from DBTP measurements, such as duration of successful one-point support (DurB), maximum posture adjustment speed, mean and maximum acceleration in the anterior-posterior (a-p) and medial-lateral (m-l) directions with eyes open and eyes closed. BW and BH were included because human motor control is directly affected by both factors. The hidden layer consists of 60 non-linear sigmoid neurons that mimics the role of the CNS by interpreting data received in the input layer. This number of neurons is chosen based on manufacturer recommendations [17]. The output layer consists of two linear neurons whose outputs are predictions of sensori-motorcontrol age and fall-probability (0 - non faller or 1 - faller, Figure 2).

Training of ANN (Figure 2(b)) requires baseline data known as inputs and targets. This data was collected from subjects aged 19 years to 60+, either with or without a history of falls. During the training process, all weights were changed iteratively through mathematical optimization (back-propagation 
algorithm16) until the inputs matched the targets. The weight matrices $\left(\mathrm{W}_{\mathrm{ij}}\right.$ and $\mathrm{W}_{\mathrm{jk}}$ ) were then fixed, resulting in a trained model that, when given measurements of new subjects, can generate their predicted age and fall possibility (Figure 2(a)).

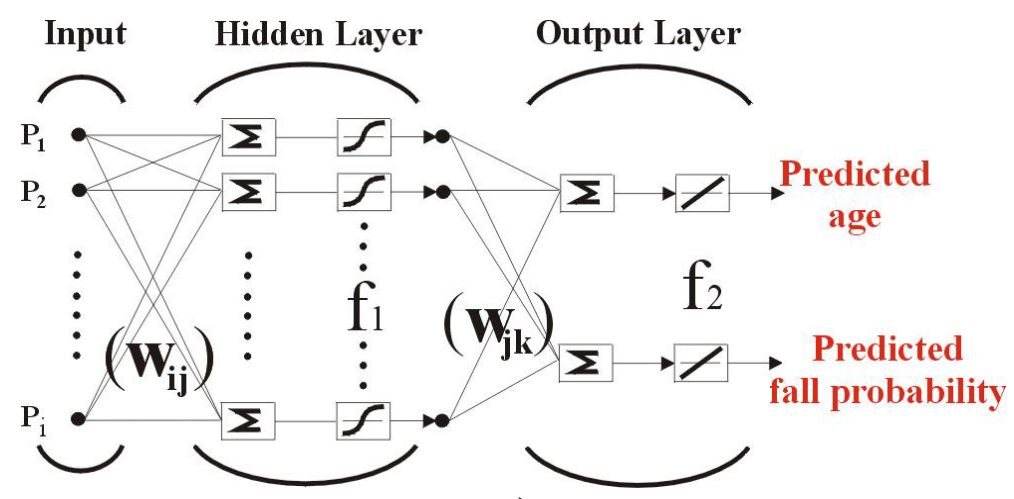

a)

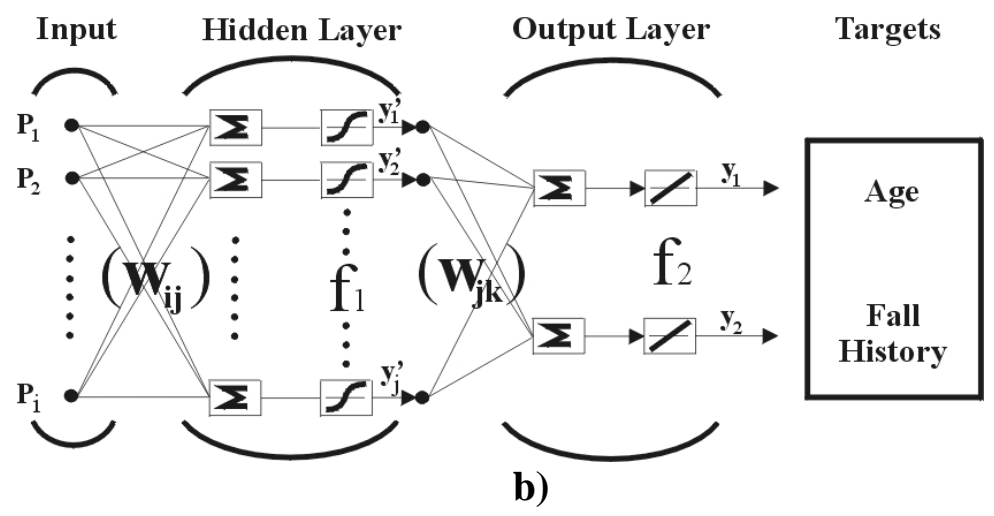

Figure 2: $\quad$ Design of the ANN model for prediction of falls and sensori-motor degeneration related to age (a) and training of the ANN model (b).

Age was designed to be an output (predicted age) because it can be an objective, easy-to-interpret criterion for judging one's state of sensori-motor function. The rate of falls increases dramatically with age regardless of one's gender, race or ethnicity [18-20]. Physical activity, however, can slow the rate of sensori-motor degeneration [18, 21]; thus, exercise can provide an individual with a "younger" body (sensori-motor-control age) than his or her true age. Contrasting one's actual age with his/her predicted sensori-motor-control age thus indicates the degree of one's sensori-motor deterioration. If the output age is higher than one's true age, for instance, the rate of degeneration is likely more rapid than that of the subjects used in the training process. 


\section{Results and discussion}

For this study, none of the subjects have a history of falls, thus the ANN model was only trained by the data of 60 non-fallers. Hence, prediction output of the model at the present state is limited to age only. Two additional subjects, a young adult and a senior, were employed to verify the validity of the model. The predicted sensori-motor-control ages were: 20 for the 22 year-old subject and 68 for the 62 year old senior.

One potential benefit of the DBTP is to evaluate any particular physical exercise, Tai Chi, for example. To do so, two ANN models are needed: model A trained by subjects with significant Tai Chi experience and model B by participants on only a minor exercise regimen. The two models can each be used to predict the sensori-motor-control age of the other model's cohort. If model A outputs younger predicted age for individuals without Tai Chi experience, then the data imply a detrimental effect of Tai Chi on sensori-motor function. Conversely, if model B outputs younger predicted age for individuals with Tai Chi experience, then the data confirm current theories [21] that Tai Chi has beneficial effects. In this manner, the cross-prediction of two different ANN models can illustrate the influence of Tai Chi on sensori-motor function. Such results could subsequently be used to guide future research on different physical exercises, particularly those designed for seniors.

Another potential benefit is to train the ANN with subjects who have fall history. After incorporating data from the subjects with a history of falls, the platform can output daily predictions of individual fall probability. Such information can be utilized to develop fall-prevention programs for seniors and could help reduce the social and health care costs associated with falls.

It should be noted that a fully trained ANN model is an integral part of the DBTP. Once connected to the platform, it will enable the device to automatically record and interpret the sensory inputs generated by the subjects. Most importantly, ANN modeling allows the DBTP to provide an assessment of sensori-motor function that the subjects themselves can understand.

\section{Conclusion}

This study demonstrated the use of simple DBTP measurements and ANN modeling technology to predict sensori-motor degradation related to age. A prototype for this promising tool was completed. We believe that, via fall prediction, this innovative device has great potentials to reduce the social and health care costs associated with age-related falls.

\section{References}

[1] Eagle DJ, Salama S, Whitman D, Evans LA, Ho E, Olde J: Comparison of three instruments in predicting accidental falls in selected inpatients in a general teaching hospital. Journal of Gerontology \& Nursing 1999, 25(7): 40-45. 
[2] Hill K, Schwarz J, Flicker L, Carroll S: Falls among healthy, communitydwelling, older women: a prospective study of frequency, circumstances, consequences and prediction accuracy. Australian and New Zealand Journal of Public Health 1999, 23(1): 41-48.

[3] Brauer SG, Burns YR, Galley P: A Prospective Study of Laboratory and Clinical Measures of Postural Stability to Predict Community-Dwelling Fallers. Journal of Gerontology: Medical Sciences 2000, 55A(8): M469M476.

[4] Burt CW, Fingerhut LA: Injury visits to hospital emergency departments: United States, 1992-95. Vital and health statistics 1998, 13(131): 1-76.

[5] Greenhouse AH: Falls among the elderly. In: Clinical neurology of aging. Edited by Albert ML. London: Oxford University Press; 1994.

[6] Sculpo TP: Falling doesn't have to be seniors' last trip. In: USA Today. vol. Nov.; 1998.

[7] Kollegger H, Baumgartner C, Wöber C, Oder W, Deecke L: Spontaneous Body Sway as a Function of Sex, Age, and Vision: Posturographic Study in 30 Healthy Adults. European Neurology 1992, 32: 253-259.

[8] Panzer VP, Bandinelli S, Hallett M: Biomechanical assessment of quiet standing and changes associated with aging. Archives of Physical Medicine and Rehabilitation 1995, 76: 151-157.

[9] Nashner LM, Peters JF: Dynamic posturography in the diagnosis and management of dizziness and balance disorders. Neurologic Clinics 1990, 8(2): 331-349.

[10] El Kashlan HK, Shepard NT, Asher AM, Wheelock SM, Telian SA: Evaluation of clinical measures of equilibrium. Laryngoscope 1998, 108(3): 311-319.

[11] Henry SM, Fung J, Horak FB: Control of stance during lateral and anterior/posterior surface translations. IEEE Transactions on Rehabilitation Engineering 1998, 6(1): 32-42.

[12] Pai YC, Maki BE, Iqbal K, McIlroy WE, Perry SD: Thresholds for step initiation induced by support-surface translation: a dynamic center-of-mass model provides much better prediction than a static model. Journal of Biomechanics 2000, 33(3): 387-392.

[13] Daubney ME, Culham EG: Lower-extremity muscle force and balance performance in adults aged 65 years and older. Physical Therapy 1999, 79(12): 1177-1185.

[14] ONeill DE, Gill KM, Krebs DE: Posturography changes do not predict functional performance changes. American Journal of Otolaryngology 1998, 19(6): 797-803.

[15] Maki BE: Biomechanical approach to quantifying anticipatory postural adjustments in the elderly. Medical \& Biological Engineering \& Computing 1993, 31(4): 355-362.

[16] Evans MK, Krebs DE: Posturography does not test vestibulospinal function. Otolaryngology - Head and Neck Surgery 1999, 120(2): 164-173.

[17] Demuth H, Beale M: Neural Network Toolbox. Natick: The MathWorks Inc; 1998. 
[18] Eagle DJ, Salama S, Whitman D, Evans LA, Ho E, Olde J: Comparison of three instruments in predicting accidental falls in selected inpatients in a general teaching hospital. Geriatrics 2001, 56: 41-45.

[19] Fuller GF: Falls in the elderly. American Family Physician 2000, 61: 21592168.

[20] Tibbits GM: Patients who fall: how to predict and prevent injuries. Geriatrics 1994, 49: 611-626.

[21] Crompton P: Tai Chi. New York: Macmilan; 1996. 